Article

\title{
Diphenyleneiodonium triggers cell death of acute myeloid leukaemia cells by blocking the mitochondrial respiratory chain and synergizes with cytarabine
}

\author{
Hassan Dakik ${ }^{1}$, Maya El Dor ${ }^{1}$, Jérôme Bourgeais ${ }^{1,2}$, Farah Kouzi ${ }^{1,3}$, Olivier Herault ${ }^{1,2}$, Fabrice \\ Gouilleux $^{1}$, Kazem Zibara ${ }^{3,4^{*}}$, Frédéric Mazurier ${ }^{{ }^{*}}$ \\ 1 University of Tours, EA7501 GICC / CNRS ERL7001 LNOx, F-37032 Tours, France \\ 2 Department of Biolocical Hematology, Tours University Hospital, F-37000 Tours, France \\ 3 Lebanese University, Faculty of Sciences, Biology Department, 90656 Beirut, Lebanon \\ 4 ER045, PRASE, 6573/14 Beirut, Lebanon \\ * Correspondence: $\underline{\text { frederic.mazurier@inserm.fr and kzibara@ul.edu.lb }}$
}

\section{Simple Summary}

Acute myeloid leukemia (AML) is an aggressive heterogeneous cancer of the blood in which $70 \%$ of cases develop relapse. Relapse is mainly due to chemoresistant leukemic cells (LCS) that are characterized by high mitochondrial oxidative phosphorylation (OxPhos) status, i.e. cells that are dependent on the mitochondrial respiratory chain $(M R C)$ function. The aim of our study was to determine whether diphenyleneiodonium (DPI), known as a potent inhibitor of flavoproteins, could be used to target AML cells. Herein, we demonstrated that DPI disrupts the mitochondrial function of AML cell lines. Interestingly, we found that cells with high-OxPhos are more sensitive to the apoptotic effects of DPI. Moreover, we showed that DPI sensitizes AML cell lines to cytarabine (Ara-C) treatment, suggesting that MRC inhibitors might be employed to target $L C$ s that are resistant to this chemotherapeutic agent.

\begin{abstract}
Acute myeloid leukaemia (AML) is characterized by the accumulation of undifferentiated blast cells in the bone marrow and blood. In most AMLs, relapse frequently occurs due to resistance to chemotherapy. Compelling research results indicate that drug resistance in cancer cells is highly dependent on the intracellular levels of reactive oxygen species (ROS). Modulating ROS levels is therefore a valuable strategy to overcome the chemotherapy resistance of leukemic cells. In this study, we evaluated the efficiency of diphenyleneiodonium (DPI), a well-known inhibitor of ROS production, in targeting AML cells. Results showed that although inhibiting cytoplasmic ROS production, DPI triggered an increase in the mitochondrial ROS levels caused by the disruption of the mitochondrial respiratory chain. We also demonstrated that DPI blocks the mitochondrial oxidative respiration (OxPhos) in a dose-dependent manner and that AML cells with high OxPhos status were highly sensitive to treatment with DPI, which synergizes with the chemotherapeutic agent cytarabine (Ara-C). Thus, our results suggest that targeting mitochondrial function by DPI might be exploited to target AML cells with high OxPhos status.
\end{abstract}

Keywords: DPI; Mitochondria; Leukaemia; Oxidative stress; OxPhos; Ara-C 


\section{Introduction}

Acute myeloid leukaemia (AML) is a heterogeneous clonal disorder of myeloid progenitors that accumulate due to a blockage in their differentiation, leading to death [1]. AML therapy has not much changed over the last decades, and more than 70\% of AML patients relapse within 3-years after therapy [2]. AML relapse is caused by residual populations of quiescent leukemic stem cells (LSCs), associated with chemoresistant AML cells that have a high mitochondrial oxidative phosphorylation $[3,4]$. Altered cellular redox status with high reactive oxygen species (ROS) levels is indeed a common hallmark of AML cells. Several lines of evidence have indicated that NOX complexes that are major contributors of ROS production, including superoxide $\left(\mathrm{O}_{2}{ }^{\bullet-}\right)$ and hydrogen peroxide $\left(\mathrm{H}_{2} \mathrm{O}_{2}\right)$, are also important regulators of AML progression and drug resistance [5,6]. Thus, targeting oxidative metabolism in AML has been proposed as a promising therapeutic strategy to eradicate AML cells[7].

We recently showed that all components of NOX2, the most prominent NOX complex across AML, are highly expressed at both the transcriptional and protein levels. Surprisingly, we did not find detectable constitutive NOX activity in 24 leukemic cell lines [8]. In addition, we demonstrated that NOX2 silencing neither affected AML cell growth nor triggered cell death in vitro. Adane et al. have recently shown that, although silencing of NOX2 induces the differentiation of primary AML cells, diphenyleneiodonium (DPI) used to inhibit NOX, did not affect the differentiation but triggered apoptosis [9]. While DPI is widely used to prove NOX activity [10], it is a non-specific inhibitor of flavoproteins that can impede the activity of nitric oxide synthases (NOS), xanthine oxidases (XOS), and complexes I and III of the mitochondrial respiratory chain (MRC) [11-13]. Importantly, DPI has been found to trigger the inhibition of the mitochondrial oxidative metabolism (OxPhos) in breast cancer cells and to induce a chemo-quiescent phenotype that blocked the propagation of cancer stem cells [14].

Recently, it has been proposed that drug resistance of AML cells might be dependent on their OxPhos status [3]. Hence, we hypothesized that DPI could target OxPhos in AML cells rather than its canonical NOX inhibition. Thus, we examined the effects of DPI on oxidative metabolism, proliferation and resistance to chemotherapy in various AML cell lines harbouring low and high OxPhos phenotypes.

\section{Results}

\subsection{DPI reduces cytoplasmic ROS, while induces superoxide production}

To study the effect of DPI on AML cells, we used eight AML cell lines, covering M0-M5 FAB stages [15] and having no endogenous NOX activity [8]. First, we measured ROS production rate using CM$\mathrm{H}_{2}$ DCFDA that detects cytoplasmic ROS (cytoROS) production, mainly $\mathrm{H}_{2} \mathrm{O}_{2}$, and dihydroethidium (DHE) taht detects intracellular superoxide $\left(\mathrm{O}_{2}{ }^{\bullet-}\right)$. We noticed a high heterogeneity in ROS production between the cell lines (Figure 1a). KG-1, HL-60, NB-4, and THP-1 cells clustered together, showing concomitant high production rates of $\mathrm{O}_{2}{ }^{\bullet-}$ and cytoROS, suggesting steady transformation of $\mathrm{O}_{2} \bullet-$ into $\mathrm{H}_{2} \mathrm{O}_{2}$. Markedly, KG-1a cells (M0), a model of immature AML, derived from $\mathrm{KG}-1$, showed low production rates of superoxide (23 RFU/min) and cytoROS (59 RFU/min). This agrees with the idea 
that more mature cells have higher ROS levels. After treatment with $20 \mu \mathrm{M}$ DPI, a dose sufficient to inhibit flavoproteins, all cell lines showed a substantial increase in $\mathrm{O}_{2}{ }^{\bullet-}$ production, while only five of them had decreased cytoROS production rates, compared to their respective controls (Figure 1b-c). To investigate the origin of $\mathrm{O}_{2}{ }^{\bullet}$ increase following DPI treatment, we measured mitochondrial $\mathrm{O}_{2}{ }^{\bullet}$ (mitoROS) production using MitoSOX, a DHE derivative that is specific to mitochondria. At steady state, the profile of $\mathrm{O}_{2}{ }^{\bullet-}$ production detected by MitoSOX was concordant to that obtained by DHE (Figure 1d). Noteworthy, KG-1a cells had the lowest level. Remarkably, DPI treatment triggered a strong induction of mitoROS levels in all cell lines (Figure 1e). Although KG-1a cells showed the strongest induction (40-fold) with DPI, its increased mitoROS level never reached the baseline levels of the other cell lines. Together, these data showed that DPI decreases cytoplasmic ROS production but concomitantly triggers an increase in mitochondrial ROS production.

a

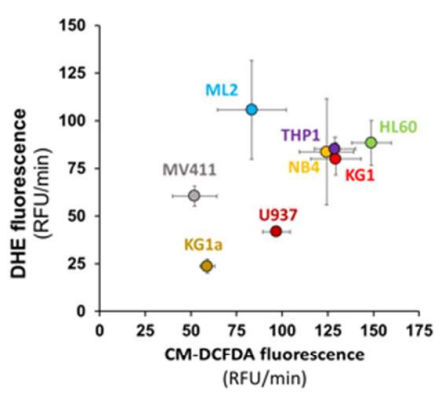

d

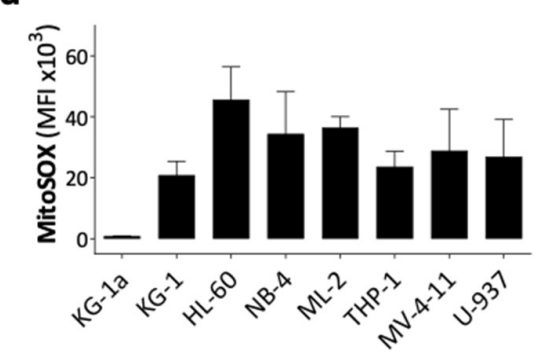

b
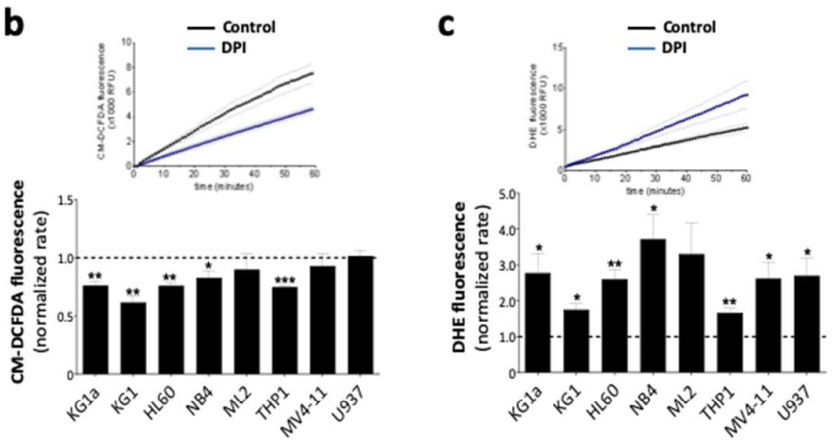

$\mathbf{e}$

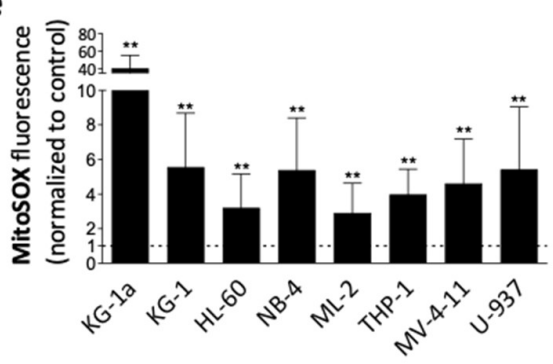

Figure 1. Steady state and DPI-induced ROS production in eight AML cell lines. (a) ROS production rates presented as DHE vs. $\mathrm{H}_{2} \mathrm{CM}$-DCFDA fluorescence rates. ROS production rate (RFU/min) was calculated from the curve's slope during $1 \mathrm{~h}$ using ClarioStar Data Analysis software. (b) Effect of DPI $(20 \mu \mathrm{M})$ on ROS production rate measured by CM-DCFDA fluorescence $(n=3)$. (c) Effect of DPI $(20 \mu \mathrm{M})$ on ROS production rate measured by DHE fluorescence $(n=3)$. DPI data are shown as normalized fluorescence rates with respect to the control for each cell line. (d-e) Baseline mitochondrial ROS production measured by MitoSox fluorescence (d). and effect of DPI on mitochondrial ROS $(e)(n=4)$. Data are shown as mean values \pm SEM. One-sample $t$-test was used to compare normalized rates to $1(* p<0.05 ; * * p<0.01 ; * * * p<0.001)$.

\subsection{DPI disrupts the mitochondrial membrane potential}

To explain the quick mitoROS burst induced by DPI, we speculated that DPI may have induced an oxidative stress by disrupting the mitochondrial respiratory chain (MRC). We thus examined the functional impact of DPI on the mitochondrial activity of AML cells by labelling with tetramethylrhodamine ethyl ester (TMRE) as a readout to determine the effects on mitochondrial membrane potential $(\Delta \Psi \mathrm{m})$. FCCP, a common mitochondrial-depolarizing agent was used as a positive control. The basal level of $\Delta \Psi \mathrm{m}$ was variable across the cell lines (Figure 2a; control black 
bars). This variability was mainly due to differences in the mitochondrial mass, as determined by Mitotracker Deep Red labelling (Figure $2 \mathrm{~b}$ and $2 \mathrm{c}$ ). DPI notably decreased $\Delta \Psi \mathrm{m}$ in all cell lines, except in KG-1a cells in which $\Delta \Psi \mathrm{m}$ was negligible, in accordance with the lowest mitochondrial biomass found in this cell line (Figure 2a, b, c). Collectively, these data indicated that DPI triggers $\mathrm{O}_{2}{ }^{\circ}$ production in AML cell lines by inhibiting MRC.

a

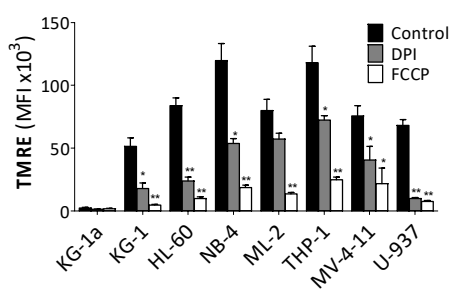

b

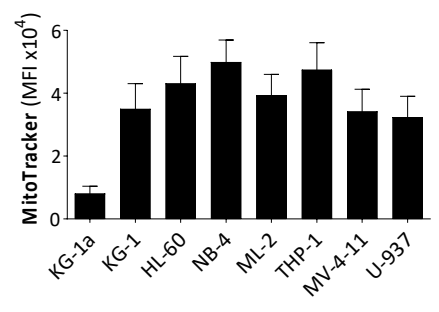

C

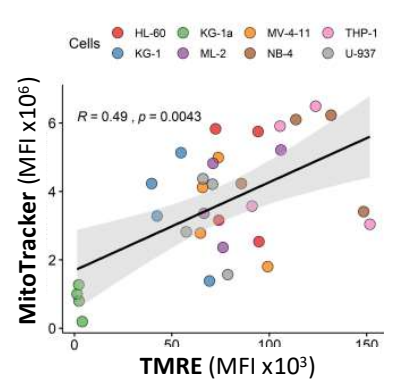

Figure 2. Effect of DPI on mitochondrial membrane potential $(\Delta \Psi \mathrm{m})$. (a) Effect of DPI on $\Delta \Psi \mathrm{m}$ was measured by TMRE fluorescence. FCCP was used as a positive control (20 uM). (b) Physiological mitochondrial mass in indicated AML cell lines was measured by MitoTracker fluorescence. (c) Spearman correlation analysis of $\Delta \Psi \mathrm{m}$ and mitochondrial mass was measured by TMRE and mitoTracker, respectively, determined in a and b. Dots represents cells from independent experiments. Data are shown as mean values \pm SEM $(n=4)$. Student's t-test was used to compare treated conditions to controls $(* p<0.05 ; * * p<0.01)$.

\subsection{DPI disrupts the MRC and alters the energetic metabolism}

To gain insights into the effect of DPI on MRC, we performed bioenergetic analyses of OxPhos through the measurement of oxygen consumption rate (OCR) using SeaHorse technology. DPI significantly reduced the OCR of six out of the eight cell lines (Figure 3a), and the maximal respiration capacity in all cell lines (Figure 3b). To examine the OCR inhibition efficiency of DPI, we calculated its IC50 values alongside the standard inhibitors antimycin A and rotenone in three representative cell lines (Figure 3c; THP-1 and MV-4-11 with high OCR, and KG-1a with low OCR). Results showed that DPI had IC50 values on OCR $(0.2-1.29 \mu \mathrm{M})$ that are in the same range as those of antimycin A $(0.26-0.53 \mu \mathrm{M})$ and rotenone $(0.55-1.22 \mu \mathrm{M})$, indicating that DPI is as efficient as current respiratory chain inhibitors (Table 1). Finally, to prove that blocking OCR may induce mitoROS, the three cell lines were treated either with DPI or a combination of rotenone and antimycin. As expected, all inhibitors triggered a similar increase in mitoROS level (Figure 2d). Together, these data indicate that DPI blocks MRC in AML cell lines and induces an oxidative burst with a similar efficiency to standard inhibitors. 
a

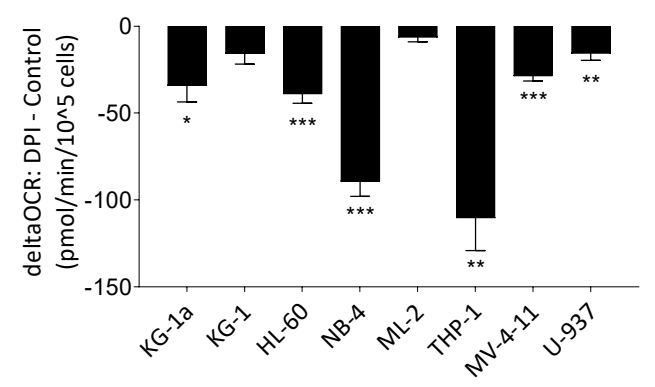

C

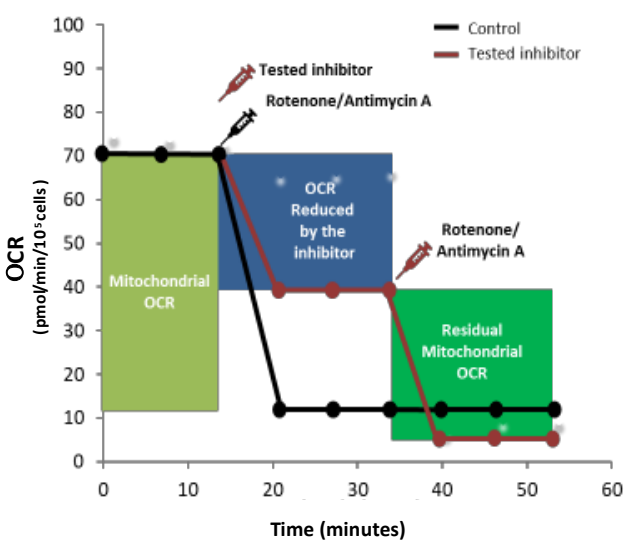

b
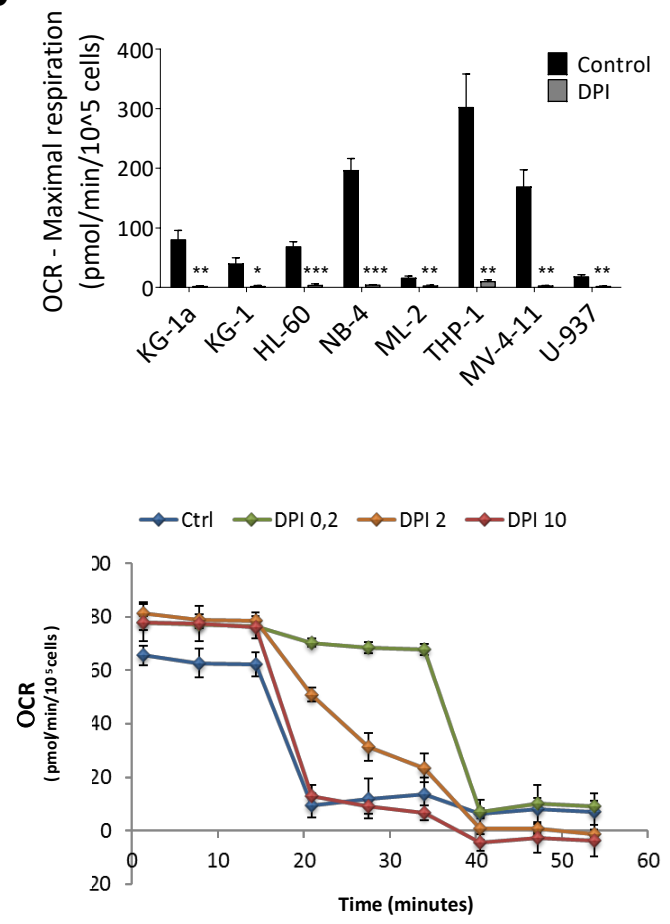

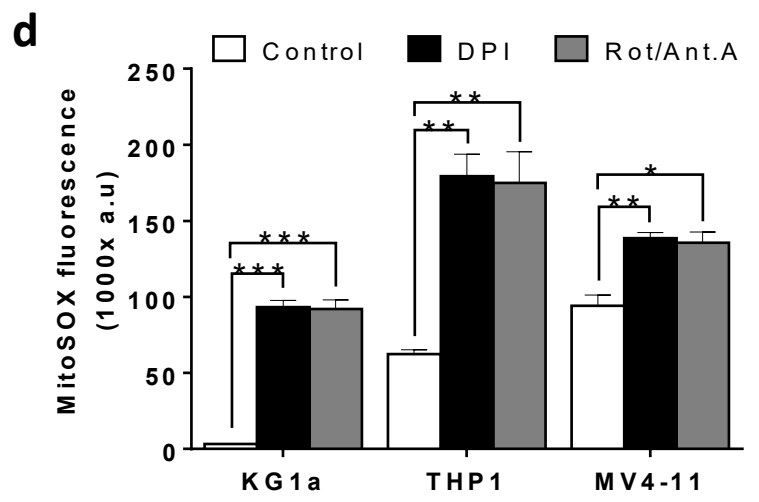

Figure 3. Effect of DPI on mitochondrial oxygen consumption rate (OCR). (a) Quantification of the acute effect of DPI on the OCR of eight AML cell lines. (b) Quantification of the effect of DPI on maximal respiration of the same cell lines. c) Left: schematic representation of the experimental design to measure the OCR IC50 values for DPI, rotenone and antimycine A. Right: a representative experiment showing OCR kinetic response of THP-1 cell line to selected DPI doses $(0.2,2$, and $10 \mu \mathrm{M})$. Mitochondrial OCR was calculated for each cell line from the control condition after injection of a mix of rotenone $(0.5 \mu \mathrm{M})$ and antimycin $\mathrm{A}(0.5 \mu \mathrm{M})$. Residual mitochondrial OCR was deduced from the inhibitor condition after a secondary injection of a mix of rotenone $(0.5 \mu \mathrm{M})$ and antimycin A $(0.5 \mu \mathrm{M})$. (d) Effect of DPI $(20 \mu \mathrm{M})$ on mitoROS in the three cell lines in comparison with rotenone $(0.5 \mu \mathrm{M})$ /antimycine $(0.5 \mu \mathrm{M})$ combination. Data are shown as mean values $\pm \mathrm{SEM}(\mathrm{n}=3)$. In (a), One-sample $\mathrm{t}$ test was used to compare delta OCR values to 0 . In (b) and (d), Student's t-test was used to compare treated conditions to controls $(* p<0.05 ; * * p<0.01 ; * * * p<0.001)$. 
Table 1. $\mathrm{IC}_{50}$ values of mitochondrial OCR for various NOX and respiratory chain inhibitors.

\begin{tabular}{cccccccc}
\hline & & \multicolumn{5}{c}{ Mitochondrial OCR IC50 } \\
\cline { 3 - 8 } & & \multicolumn{2}{c}{ KG1a } & \multicolumn{2}{c}{ THP1 } & MV-4-11 \\
\cline { 3 - 8 } Inhibitors & Target & $\mathrm{IC}_{50}(\mu \mathrm{M})$ & $95 \% \mathrm{CI}(\mu \mathrm{M})$ & $\mathrm{IC}_{50}(\mu \mathrm{M})$ & $95 \% \mathrm{CI}(\mu \mathrm{M})$ & $\mathrm{IC}_{50}(\mu \mathrm{M})$ & $95 \% \mathrm{CI}(\mu \mathrm{M})$ \\
\hline Rotenone & Complex I & 0.55 & 0.40 to 0.76 & 1.22 & 0.83 to 1.80 & 0.64 & 0.51 to 0.82 \\
Antimycin A & Complex III & 0.26 & 0.18 to 0.39 & 0.53 & 0.40 to 0.69 & 0.32 & 0.24 to 0.42 \\
DPI & Flavoproteins & 0.2 & 0.13 to 0.30 & 1.29 & 0.91 to 1.82 & 0.78 & 0.55 to 1.11 \\
\hline
\end{tabular}

Note: $\mathrm{IC}_{50}$ values (micromolars) are calculated by a nonlinear fit model and shown as estimated value with $95 \%$ CI. Abbreviations: $\mathrm{IC}_{50}, 50 \%$ inhibitory concentration. CI, confidence interval.

\subsection{DPI reduces cell proliferation and triggers apoptosis}

Since DPI induced oxidative stress by blocking mitochondrial respiration, we examined its impact on cell growth and survival. To address the effect of a chronic exposure, we used a low dose of DPI $(0.2 \mu \mathrm{M})$ and followed the proliferation of AML cell lines for three days. The results showed that all cell lines exposed to DPI had a significant reduction in their expansion capacity compared to their corresponding controls (Figure 4a). Furthermore, to investigate whether reduced expansion resulted from proliferation slowdown or cell death induction, we quantified apoptosis at day 3 of culture, using Annexin-V and 7-AAD (Figure 4b). Following DPI treatment, most cell lines showed moderate to high levels of apoptosis that could partially explain cell growth reduction (Figure 4a-b). NB-4, THP-1, and MV-4-11 cells, which showed the highest apoptosis rates (Figure 4b), were also those with the highest maximal OCR capacities (Figure 3b). In contrast, KG-1a cells that showed low mitoROS and minimal $\Delta \Psi \mathrm{m}$ (Figures $1 \mathrm{~d}$ and $2 \mathrm{a}$ ) also showed minimal apoptosis (Figure $4 \mathrm{~b}$ ). Together, these data suggest that DPI reduces cell growth by inhibiting cell division in an apoptosis-dependent manner and that cells with high OxPhos metabolism are more sensitive to DPI-induced apoptosis. 
a

- Control $\rightarrow$ DPI
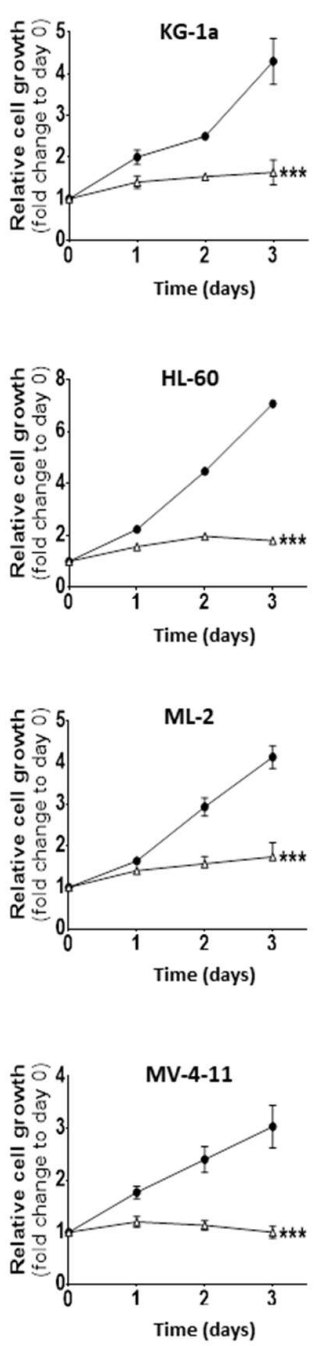
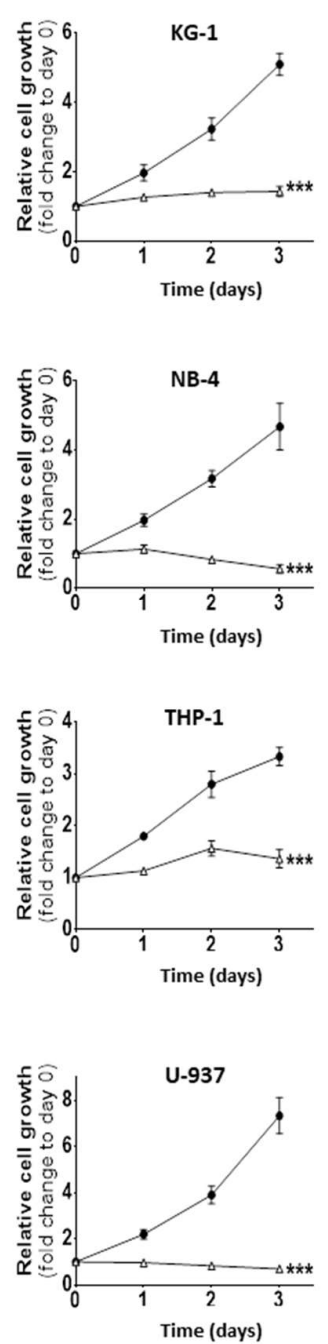

b

$\square$ Live $\square$ Early apoptotic $\square$ Late apoptotic
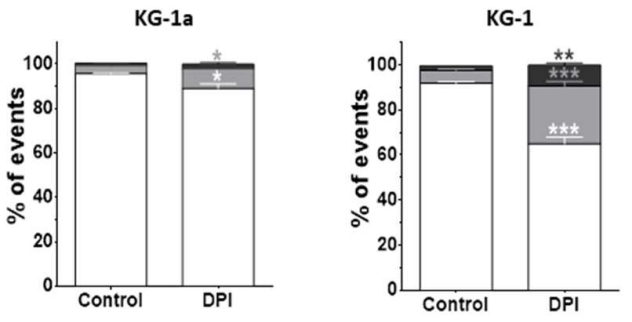

HL-60
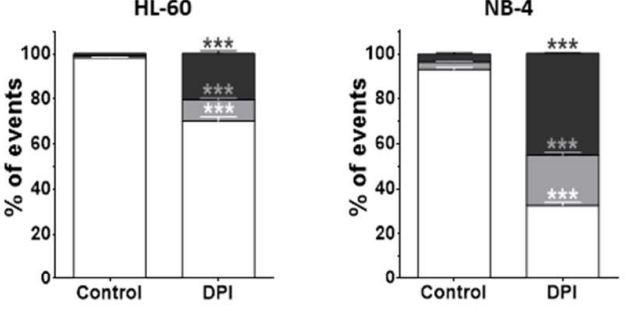

ML-2

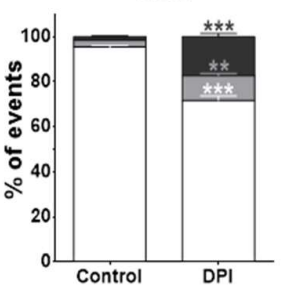

THP-1

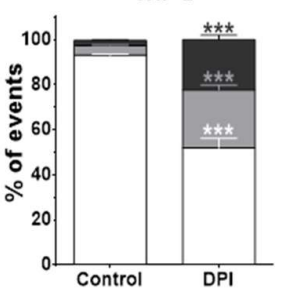

MV-4-11

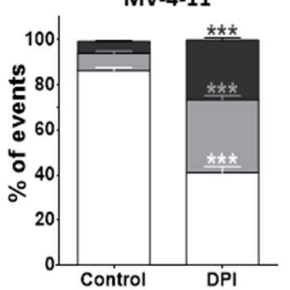

U-937

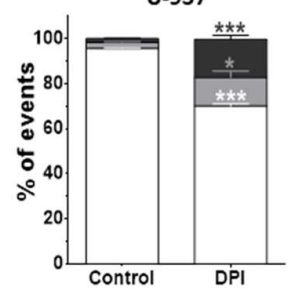

Figure 4. Effect of DPI on cell proliferation and apoptosis. Cell growth was assessed using resazurin reduction assay at indicated days, following DPI $(0.2 \mu \mathrm{M})$ treatment. Analysis of apoptosis was performed at day 3 after treatment. (a) Growth curves from various conditions for eight AML cell lines. Relative cell growth was calculated as resazurin fluorescence fold change compared to control at day 0 . Data are shown as mean \pm SEM $(n=3)$. Two-way ANOVA was performed for each cell line followed by Tukey's post hoc analysis. Adjusted $p$ values are shown from day 3 comparing DPI condition to DMSO control $(* \mathrm{p}<0.05 ; * * \mathrm{p}<0.01 ; * * * \mathrm{p}<0.001)$. (b) Apoptosis in DPI-treated AML cell lines. 7-AAD/Annexin V staining distinguishes between live, early apoptotic and late apoptotic cells. Data are shown as mean $\pm S E M(n=3$ independent experiments). Student's ttest was used to compare DPI conditions to their corresponding control counterparts $(* p<0.05 ; * * p<0.01 ; * * *$ $p<0.001)$.

\subsection{Effect of combination therapy of DPI and cytarabin on AML cell lines}

Recent findings have suggested that AML cells with high OxPhos are more resistant to therapeutic agents [3,4]. Therefore, we investigated whether DPI may synergize with Ara-C to eliminate AML cells. To address this issue, we used two representative cell lines, THP-1 and MV-4-11, with high OxPhos, and KG-1a, with the lowest OxPhos status. A dose-response matrix was designed to test 35 different combinations of doses ranging from 0 to $0.5 \mu \mathrm{M}$ for Ara-C and 0 to $0.4 \mu \mathrm{M}$ for DPI (Figure 
5a). Data showed that the combination of DPI and Ara-C had synergistic effect in THP-1 and MV-411 cells (positive Loewe scores) but not in KG-1a (a negative score) (Figure 5a-b). This suggests that only the cell lines with high OCR might be sensitized by DPI.

a

KG-1a

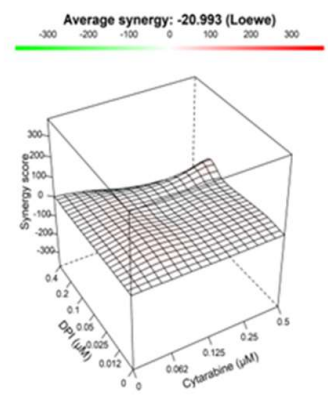

b

KG-1a
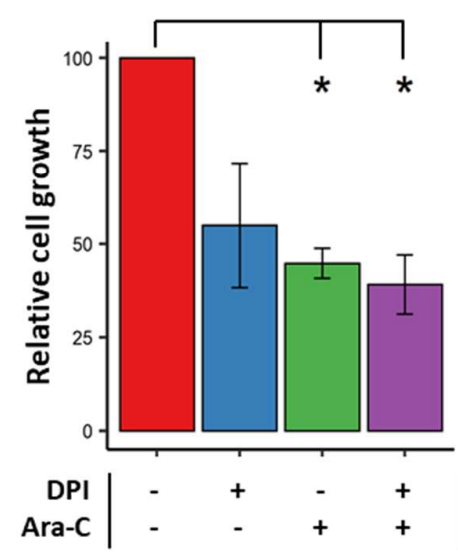

MV-4-11

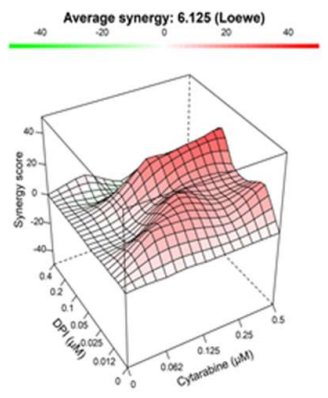

MV-4-11

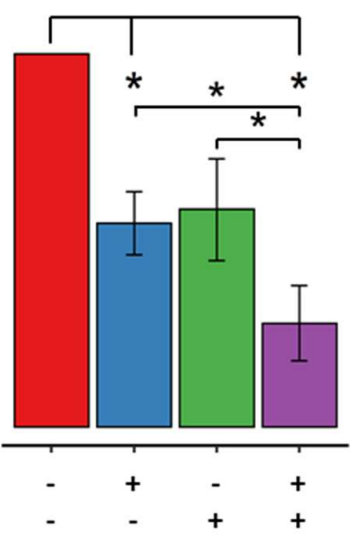

THP-1

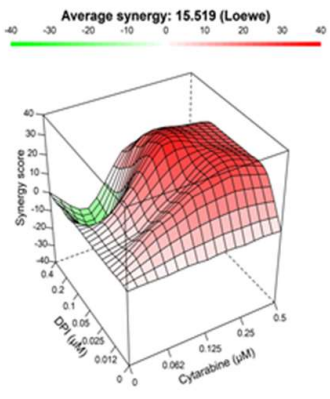

THP-1

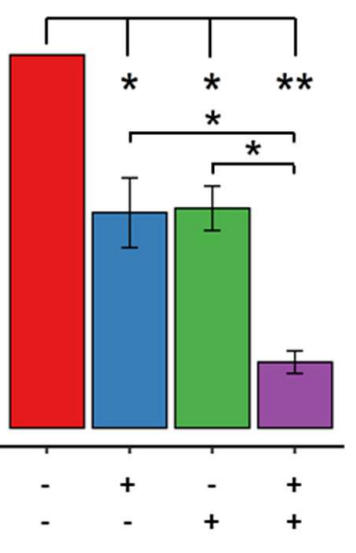

Figure 5. Effect of combination therapy of DPI and Ara-C. (a) 3D interaction landscape showing Loewe's synergy score for the combination of various doses of DPI and Ara-C on growth inhibition of KG-1a, THP1 and MV-4-11 cell lines. Synergy score on $\mathrm{Z}$ axis corresponds to excess $\%$ inhibition beyond the expectation by the Loewe additivity equation. This score reflects the percentage of growth inhibition that can be attributed to the drug interactions. An average synergy score is shown over the dose-response matrix. Data are shown as the average from 3 independent experiments. (b) Barplots showing the effects of DPI (0.1 $\mu \mathrm{M})$, Ara-C $(0.25 \mu \mathrm{M})$ or their combination on cell growth, as compared to control. Data are shown as mean values \pm SEM $(\mathrm{n}=3)$. Student's t-test followed by BH adjustment was used for pairwise comparisons $\left({ }^{*} p\right.$ $\left.<0.05 ;{ }^{* *} p<0.01\right)$.

\section{Discussion}

This study aimed to understand the mode of action of DPI in AML cell lines. We demonstrated that DPI, although inhibiting cytoplasmic ROS production, disrupts MRC, increases mitochondrial ROS production, and triggers the apoptosis of AML cell lines, especially those with a high-OxPhos status. In addition, we showed that DPI synergizes with Ara-C in eliminating high-OxPhos AML cells preferentially. 
First, we showed that DPI reduces cytoROS production in five of the eight tested cell lines. This decrease was previously shown in three leukemic cell lines (KU-812, MOLM-13 and HEL) treated with DPI [16]. DPI had also been found to reduce the cytoROS production, thereby impacting the proliferation of prostate cancer cells [17]. Moreover, our results showed that DPI can inhibit the mitochondrial respiration, induce a rise in mitoROS level and induce apoptosis. This is in line with the data showing that DPI induces cell cycle arrest and decreases the mitochondrial potential of prostate cancer cell lines [18]. Furthermore, Ozsvari et al. demonstrated that the treatment of breast cancer cell lines with DPI inhibited mitochondrial oxidative metabolism (OxPhos), thereby reducing mitochondrial ATP production by more than 90\% [14]. However, DPI did not trigger the production of mitochondrial ROS in these cells, which could be explained by the use of a very low dose (10 nM). Collectively, these data showed that DPI can efficiently inhibit mitochondrial respiration in different types of cancer cells even at very low doses.

Our data indicated that DPI can readily impede the mitochondrial respiration in OxPhos-high AML cells, independently of NOX inhibition, causing a strong burst of superoxide production. Moreover, we recently reported that another NOX inhibitor, VAS3947, induces the apoptosis of AML cells through cysteine thiol alkylation, independently of NOX inhibition [19]. Several studies indicated that NOX complexes are important regulators of AML progression and drug resistance [9,20-25], but many of these have used non-specific inhibitors, including DPI and VAS3947, to prove NOX activity or to study the functional impact of NOX inhibition on leukemic cells. Our findings suggest that the use of such inhibitors to study the role of NOX in oxidative metabolism can be misleading and highlight the need to develop more specific NOX inhibitors.

We showed for the first time that DPI acts synergistically with Ara-C to induce apoptosis in AML cells having a high-OxPhos metabolism (THP-1 and MV-4-11), but not in low-OxPhos cells (KG-1a). The combined treatment of DPI with FLT3-ITD inhibitors, midostaurin or sorafenib, has been found to synergistically inhibit the proliferation of AML cell lines harbouring FLT3-ITD, and its combination with the tyrosine kinase inhibitor imatinib has been showed to synergistically increase apoptosis in chronic myeloid leukemia (CML) cells in vivo [25]. Remarkably, the authors have demonstrated that the viability of healthy CD34-positive cells has not been affected by DPI, suggesting that this compound might be safely used in the treatment of myeloid leukaemias. Recent reports revealed that chemoresistance and relapse may arise from cells bearing high-OxPhos metabolism [3,4]. Notably, Ara-C resistant AML populations exhibit metabolic characteristics and gene signatures compatible with a high-OxPhos status [3]. In these cells, targeting the mitochondrial metabolism induced an energetic shift towards low-OxPhos and enhanced anti-leukaemic effects of Ara-C [3]. Altogether, these data support our findings that targeting the high-OxPhos status of AML cells might help to overcome the resistance to chemotherapy.

In summary, this work reports that DPI antileukemic activity is caused by the inhibition of MRC and OxPhos disruption. We also found that DPI can synergize with Ara-C in targeting high-OxPhos AML cells. Thus, our data pave the development of therapies that specifically target mitochondrial respiration in myeloid leukaemias in the future. 


\section{Materials and Methods}

\subsection{Cell lines and culture}

Human myeloid leukaemia cell lines (KG-1a, KG-1, HL-60, NB-4, ML-2, THP-1, MV-4-11, U-937) were purchased from DSMZ (German Collection of Microorganisms \& Cell Cultures, Braunschweig, Germany). Cells were cultured in RPMI media (Life Technologies, Villebon-sur-Yvette, France) supplemented with $10 \%$ heat-inactivated fetal bovine serum (FBS, Life Technologies), 2 mM Lglutamine (Life Technologies), $100 \mathrm{U} / \mathrm{mL}$ penicillin (Sigma-Aldrich, Saint-Quentin-Fallavier, France) and $100 \mu \mathrm{g} / \mathrm{mL}$ streptomycin (Boehringer-Mannheim, Meylan, France) at $37^{\circ} \mathrm{C}$ in fully humidified air and $5 \% \mathrm{CO}_{2}$. For all experiments, cells were harvested from culture while in their exponential growth phase.

\subsection{ROS measurement}

\subsubsection{Fluorometric assay(CM-DCFDA and DHE)}

Cells were washed with PBS and seeded in a 96-well plate at $2.10^{5}$ cells per well in a $200 \mu \mathrm{L}$ reaction volume in the presence or absence of DPI $(20 \mu \mathrm{M})$ (Sigma-Aldrich, Saint-Quentin-Fallavier, France). DMSO was used as vehicle control. Cytoplasmic ROS and intracellular superoxide productions were detected by 5-(and-6)-chloromethyl-2',7'-dichlorodihydrofluorescein diacetate (CM-DCFDA) (Life Technologies) or Dihydroethidium (DHE) (Invitrogen, Villebon sur Yvette, France), respectively, each with a concentration of $5 \mu \mathrm{M}$ per well. ROS production was measured kinetically for CMDCFDA $(\lambda \mathrm{ex}=483-14 \mathrm{~nm}, \lambda \mathrm{em}=530-30 \mathrm{~nm})$ and DHE $\left(\lambda_{\mathrm{ex}}=490-15 \mathrm{~nm}, \lambda_{\mathrm{em}}=600-20 \mathrm{~nm}\right)$ every minute for $1 \mathrm{~h}$ at $37^{\circ} \mathrm{C}$ using a ClarioStar plate reader (BMG Labtech, Champigny-sur-Marne, France). ROS production rate (RFU/min) was calculated via the ClarioStar Data Analysis software using the slope of the curve at steady state.

\subsubsection{Flow cytometry assay (MitoSOX)}

Cells were washed with PBS and resuspended at $1.10^{5}$ cells in $100 \mu \mathrm{L}$ reaction volume in the presence or absence of DPI (20 $\mu \mathrm{M})$. DMSO was used as vehicle control. Mitochondrial superoxide production was measured using MitoSOX (Life Technologies), which was added at $5 \mu \mathrm{M}$ per condition. The samples were incubated in the dark for $30 \mathrm{~min}$ at $37^{\circ} \mathrm{C}$ prior to fluorescence measurement on a $\mathrm{C} 6$ Accuri ${ }^{\circledR}$ flow cytometer (Becton Dickinson, Le Pont de Claix, France). Data were analysed using FlowJo® software (Becton Dickinson).

\subsection{Mitochondrial function measurement}

\subsubsection{Mitochondrial mass}

The relative quantification of mitochondrial mass was evaluated using the fluorescent probe MitoTracker Deep Red (Invitrogen) according to the supplier's instructions. Briefly, cells $\left(1.10^{5}\right)$ were harvested from culture and then stained with MitoTracker (50 nM) in $200 \mu \mathrm{L}$ of fresh RPMI media without FBS for $30 \mathrm{~min}$ at $37^{\circ} \mathrm{C}$ in the dark. Cells were then washed in warm PBS and fluorescence 
intensity was measured by flow cytometry on C6 Accuri® flow cytometer. DMSO was used as a vehicle control. FlowJo® Software ${ }^{\circledR}$ was used for data analysis.

\subsubsection{Mitochondrial membrane potential}

Mitochondrial membrane potential $(\Delta \Psi \mathrm{m})$ was assessed using the fluorescent probe tetramethylrhodamine ethyl ester (TMRE; Invitrogen). A total of $1.10^{5}$ cells were resuspended in fresh RPMI media supplemented with 10\% FBS and stained with TMRE (100 nM) in presence or absence of DPI $(20 \mu \mathrm{M})$ in $200 \mu \mathrm{L}$ for $30 \mathrm{~min}$ at $37^{\circ} \mathrm{C}$ in the dark. Cells were then washed in warm PBS and fluorescence intensity was measured by flow cytometry on C6 Accuri ${ }^{\circledR}$ flow cytometer. FCCP $(20 \mu \mathrm{M})$ (Selleckchem Houston, USA) was used as positive control and DMSO was used as vehicle control. FlowJo® Software ${ }^{\circledR}$ was used for data analysis.

\subsection{Mitochondrial respiration}

Oxygen consumption rate (OCR) was quantified using a Seahorse XFe96 Analyzer (Agilent Technologies), as described previously with slight modifications [26]. Briefly, cells were plated at $1.10^{5}$ cells per well in XF96 (Agilent) cell culture plates. OCR measurements were carried in a substrate-free base medium supplemented with $2 \mathrm{mM}$ glutamine (Gibco, Carlsba, CA, USA) and $10 \mathrm{mM}$ glucose (Sigma-Aldrich). The OCR values are presented as pmoles $/ \mathrm{min} / 10^{5}$ cells. Sequential injections of DPI (20 $\mu \mathrm{M}$; Sigma) or vehicle DMSO, oligomycin (1 $\mu \mathrm{M}$; Sigma), DNP (100 $\mu \mathrm{M})$, and rotenone/antimycin A (0.5 $\mu \mathrm{M}$; Sigma) were used to determine the main respiratory parameters, in particular the acute response to DPI and the impact of the latter on maximal respiration. A modified strategy was used to calculate IC50 values for DPI, rotenone and antimycine A, as described in Figure $2 \mathrm{~d}$. The effect of DPI, or standard inhibitors, concentrations on the OCR variation were compared with the effect of the rotenone antimycin A mix, used as a positive control for inhibition of the respiratory chain. This allowed to define the IC50 of the respiratory activity for each compound.

\subsection{Apoptosis assay}

Cells were cultured alone or in the presence of DPI $(0.2 \mu \mathrm{M})$. Three days following drug addition, cells were harvested and washed with cold PBS and then resuspended in Annexin V Binding Buffer (BioLegend, London, UK). Next, the cells were stained with APC-conjugated Annexin V (BioLegend) and 7-AAD (Sigma-Aldrich) according to BioLegend's instructions and then analysed using C6 Accuri ${ }^{\circledR}$ flow cytometer and FlowJo® software.

\subsection{Drug combination assay}

Cells were seeded in $160 \mu \mathrm{L}$ media at a density of $4 \times 10^{3}$ cells/well and incubated overnight at $37^{\circ} \mathrm{C}$. They were then exposed to various concentrations of DPI and Cytarabine (Ara-C) (Sandoz France Levallois-Perret, France) in a final volume of $200 \mu \mathrm{L}$. Proliferation assay was followed after $72 \mathrm{~h}$ of treatment through resazurin fluorescence assay. Resazurin $(0.1 \mathrm{mg} / \mathrm{mL})$ was added at $20 \mu \mathrm{L} /$ well and incubated for $4 \mathrm{~h}$ at $37^{\circ} \mathrm{C}$ in the dark, then fluorescence was $\left(\lambda_{\mathrm{ex}}=529.5-19 \mathrm{~nm}, \lambda_{\mathrm{em}}=582-36 \mathrm{~nm}\right)$ measured using ClarioStar microplate reader. Synergy analyses was done in R environment using the Synergyfinder package [27]. 


\section{Conclusions}

This work 1) demonstrates that DPI affects AML proliferation in absence of NOX activity; 2) confirms its inhibitory effect on MRC, and 3) shows that combining conventional chemotherapy with a MRC inhibitor may help to eradicate the chemotherapy resistance of leukemic cells.

Author Contributions: HD, KZ and FM: Conceptualization. HD, MED, JB, FK: Methodology and Investigation. HD, MED, JB, FK, FG, OH, KZ and FM: Formal analysis. KZ and FM: Supervision. HD, KZ, FG and FM: Writing - original draft. FM: Project administration. KZ and FM: funding acquisition. All authors: Writing - Review and editing. All authors have read and agreed to the published version of the manuscript.

Funding: HD received grants from the Lebanese south governate and the French "Fondation ARC pour la recherche sur le cancer". MD received a grant from the Lebanese south governate. This work was supported by grants from "the French Committees of the "Ligue Contre le Cancer Grand-Ouest" [36 (Indre), 37 (Indre-etLoire), and 41 (Loir et Cher)] (FM), the Hubert Curien Program (CEDRE) (FM, KZ), the Lebanese University (KZ), and the Lebanese National Council for Scientific Research (KZ).

Acknowledgments: In this section you can acknowledge any support given which is not covered by the author contribution or funding sections. This may include administrative and technical support, or donations in kind (e.g., materials used for experiments).

Conflicts of Interest: The authors declare no conflict of interest.

\section{References}

1. Dohner, H.; Estey, E.; Grimwade, D.; Amadori, S.; Appelbaum, F.R.; Buchner, T.; Dombret, H.; Ebert, B.L.; Fenaux, P.; Larson, R.A.; et al. Diagnosis and management of AML in adults: 2017 ELN recommendations from an international expert panel. Blood 2017, 129, 424-447, doi:10.1182/blood-201608-733196.

2. Gregory, T.K.; Wald, D.; Chen, Y.C.; Vermaat, J.M.; Xiong, Y.; Tse, W. Molecular prognostic markers for adult acute myeloid leukemia with normal cytogenetics. Journal of Hematology \& Oncology 2009, 2, 10, doi:10.1186/1756-8722-2-23.

3. Farge, T.; Saland, E.; de Toni, F.; Aroua, N.; Hosseini, M.; Perry, R.; Bosc, C.; Sugita, M.; Stuani, L.; Fraisse, M.; et al. Chemotherapy-Resistant Human Acute Myeloid Leukemia Cells Are Not Enriched for Leukemic Stem Cells but Require Oxidative Metabolism. Cancer Discovery 2017, 7, 716-735, doi:10.1158/2159-8290.cd-16-0441.

4. Boyd, A.L.; Aslostovar, L.; Reid, J.; Ye, W.; Tanasijevic, B.; Porras, D.P.; Shapovalova, Z.; Almakadi, M.; Foley, R.; Leber, B.; et al. Identification of Chemotherapy-Induced Leukemic-Regenerating Cells Reveals a Transient Vulnerability of Human AML Recurrence. Cancer Cell 2018, 34, 483-+, doi:10.1016/j.ccell.2018.08.007.

5. Herault, O.; Hope, K.J.; Deneault, E.; Mayotte, N.; Chagraoui, J.; Wilhelm, B.T.; Cellot, S.; Sauvageau, M.; Andrade-Navarro, M.a.; Hébert, J.; et al. A role for GPx3 in activity of normal and leukemia stem cells. The Journal of experimental medicine 2012, 209, 895-901, doi:10.1084/jem.20102386.

6. Lagadinou, E.D.; Sach, A.; Callahan, K.; Rossi, R.M.; Neering, S.J.; Minhajuddin, M.; Ashton, J.M.; Pei, S.S.; Grose, V.; O'Dwyer, K.M.; et al. BCL-2 Inhibition Targets Oxidative Phosphorylation and Selectively Eradicates Quiescent Human Leukemia Stem Cells. Cell stem cell 2013, 12, 329-341, doi:10.1016/j.stem.2012.12.013. 
7. Trombetti, S.; Cesaro, E.; Catapano, R.; Sessa, R.; Lo Bianco, A.; Izzo, P.; Grosso, M. Oxidative Stress and ROS-Mediated Signaling in Leukemia: Novel Promising Perspectives to Eradicate Chemoresistant Cells in Myeloid Leukemia. International Journal of Molecular Sciences 2021, 22, doi:10.3390/ijms22052470.

8. Dakik, H.; El Dor, M.; Leclerc, J.; Kouzi, F.; Nehme, A.; Deynoux, M.; Debeissat, C.; Khamis, G.; Ducrocq, E.; Ibrik, A.; et al. Characterization of NADPH Oxidase Expression and Activity in Acute Myeloid Leukemia Cell Lines: A Correlation with the Differentiation Status. Antioxidants 2021, 10, doi:10.3390/antiox10030498.

9. $\quad$ Adane, B.; Ye, H.B.; Khan, N.; Pei, S.; Minhajuddin, M.; Stevens, B.M.; Jones, C.L.; D'Alessandro, A.; Reisz, J.A.; Zaberezhnyy, V.; et al. The Hematopoietic Oxidase NOX2 Regulates Self-Renewal of Leukemic Stem Cells. Cell Reports 2019, 27, 238-+, doi:10.1016/j.celrep.2019.03.009.

10. Cifuentes-Pagano, E.; Meijles, D.N.; Pagano, P.J. The Quest for Selective Nox Inhibitors and Therapeutics: Challenges, Triumphs and Pitfalls. Antioxidants \& Redox Signaling 2014, 20, 2741-2754, doi:10.1089/ars.2013.5620.

11. Stuehr, D.J.; Fasehun, O.A.; Kwon, N.S.; Gross, S.S.; Gonzalez, J.A.; Levi, R.; Nathan, C.F. INHIBITION OF MACROPHAGE AND ENDOTHELIAL-CELL NITRIC-OXIDE SYNTHASE BY DIPHENYLENEIODONIUM AND ITS ANALOGS. Faseb Journal 1991, 5, 98-103.

12. Li, Y.B.; Trush, M.A. Diphenyleneiodonium, an NAD $(\mathrm{P}) \mathrm{H}$ oxidase inhibitor, also potently inhibits mitochondrial reactive oxygen species production. Biochemical and Biophysical Research Communications 1998, 253, 295-299, doi:10.1006/bbrc.1998.9729.

13. Lambert, A.J.; Buckingham, J.A.; Boysen, H.M.; Brand, M.D. Diphenyleneiodonium acutely inhibits reactive oxygen species production by mitochondrial complex I during reverse, but not forward electron transport. Biochimica et Biophysica Acta (BBA) - Bioenergetics 2008, 1777, 397-403, doi:10.1016/j.bbabio.2008.03.005.

14. Ozsvari, B.; Bonuccelli, G.; Sanchez-Alvarez, R.; Foster, R.; Sotgia, F.; Lisanti, M.P. Targeting flavincontaining enzymes eliminates cancer stem cells (CSCs), by inhibiting mitochondrial respiration: Vitamin B2 (Riboflavin) in cancer therapy. Aging-Us 2017, 9, 2610-2628, doi:10.18632/aging.101351.

15. Bennett, J.M.; Catovsky, D.; Daniel, M.T.; Flandrin, G.; Galton, D.A.G.; Gralnick, H.R.; Sultan, C. PROPOSALS FOR CLASSIFICATION OF ACUTE LEUKEMIAS. British Journal of Haematology 1976, 33, 451-\&, doi:10.1111/j.1365-2141.1976.tb03563.x.

16. Reddy, M.M.; Fernandes, M.S.; Salgia, R.; Levine, R.L.; Griffin, J.D.; Sattler, M. NADPH oxidases regulate cell growth and migration in myeloid cells transformed by oncogenic tyrosine kinases. Leukemia 2011, 25, 281-289, doi:10.1038/leu.2010.263.

17. Brar, S.S.; Corbin, Z.; Kennedy, T.P.; Hemendinger, R.; Thornton, L.; Bommarius, B.; Arnold, R.S.; Whorton, A.R.; Sturrock, A.B.; Huecksteadt, T.P.; et al. NOX5 NAD(P)H oxidase regulates growth and apoptosis in DU 145 prostate cancer cells. American Journal of Physiology - Cell Physiology 2003, 285.

18. Kumar, B.; Koul, S.; Khandrika, L.; Meacham, R.B.; Koul, H.K. Oxidative stress is inherent in prostate cancer cells and is required for aggressive phenotype. Cancer Research 2008, 68, 1777-1785, doi:10.1158/0008-5472.can-07-5259.

19. El Dor, M.; Dakik, H.; Polomski, M.; Haudebourg, E.; Brachet, M.; Gouilleux, F.; Prie, G.; Zibara, K.; Mazurier, F. VAS3947 Induces UPR-Mediated Apoptosis through Cysteine Thiol Alkylation in AML Cell Lines. Int. J. Mol. Sci. 2020, 21, doi:10.3390/ijms21155470.

20. Hole, P.S.; Zabkiewicz, J.; Munje, C.; Newton, Z.; Pearn, L.; White, P.; Marquez, N.; Hills, R.K.; Burnett, A.K.; Tonks, A.; et al. Overproduction of NOX-derived ROS in AML promotes proliferation and is 
associated with defective oxidative stress signaling. Blood 2013, 122, 3322-3330, doi:10.1182/blood-201304-491944.

21. Marlein, C.R.; Zaitseva, L.; Piddock, R.E.; Robinson, S.D.; Edwards, D.R.; Shafat, M.S.; Zhou, Z.; Lawes, M.; Bowles, K.M.; Rushworth, S.A. NADPH oxidase-2 derived superoxide drives mitochondrial transfer from bone marrow stromal cells to leukemic blasts. Blood 2017, 130, 1649-1660, doi:10.1182/blood-2017-03-772939.

22. Jayavelu, A.K.; Moloney, J.N.; Böhmer, F.-D.; Cotter, T.G. NOX-driven ROS formation in cell transformation of FLT3-ITD positive AML. Experimental Hematology 2016, doi:10.1016/j.exphem.2016.08.008.

23. Mondet, J.; Lo Presti, C.; Garrel, C.; Skaare, K.; Mariette, C.; Carras, S.; Park, S.; Carre, M.; Bulabois, C.E.; Molina, L.; et al. Adult patients with de novo acute myeloid leukemia show a functional deregulation of redox balance at diagnosis which is correlated with molecular subtypes and overall survival. Haematologica 2019, 104, doi:10.3324/haematol.2018.206821.

24. Robinson, A.J.; Hopkins, G.L.; Rastogi, N.; Hodges, M.; Doyle, M.; Davies, S.; Hole, P.S.; Omidvar, N.; Darley, R.L.; Tonks, A. Reactive Oxygen Species Drive Proliferation in Acute Myeloid Leukemia via the Glycolytic Regulator PFKFB3. Cancer Research 2020, 80, 937-949, doi:10.1158/0008-5472.can-19-1920.

25. Sanchez-Sanchez, B.; Gutierrez-Herrero, S.; Lopez-Ruano, G.; Prieto-Bermejo, R.; Romo-Gonzalez, M.; Llanillo, M.; Pandiella, A.; Guerrero, C.; Miguel, J.F.S.; Sanchez-Guijo, F.; et al. NADPH oxidases as therapeutic targets in chronic myelogenous leukemia. Clinical cancer research : an official journal of the American Association for Cancer Research 2014, 20, 4014-4025, doi:10.1158/1078-0432.ccr-13-3044.

26. Kouzi, F.; Zibara, K.; Bourgeais, J.; Picou, F.; Gallay, N.; Brossaud, J.; Dakik, H.; Roux, B.; Hamard, S.; Le Nail, L.R.; et al. Disruption of gap junctions attenuates acute myeloid leukemia chemoresistance induced by bone marrow mesenchymal stromal cells. Oncogene 2020, 39, 1198-1212, doi:10.1038/s41388019-1069-y.

27. He, L.; Kulesskiy, E.; Saarela, J.; Turunen, L.; Wennerberg, K.; Aittokallio, T.; Tang, J. Methods for Highthroughput Drug Combination Screening and Synergy Scoring. Methods in molecular biology (Clifton, N.J.) 2018, 1711, 351-398, doi:10.1007/978-1-4939-7493-1_17.

Publisher's Note: MDPI stays neutral with regard to jurisdictional claims in published maps and institutional affiliations.

(C) 2020 by the authors. Submitted for possible open access publication under the terms and conditions of the Creative Commons Attribution (CC BY) license (http://creativecommons.org/licenses/by/4.0/). 